\title{
Letter to the Editor: The Role of the Annals of Geriatric Medicine and Research as a Platform for Validating Smart Healthcare Devices for Older Adults
}

In the midst of the undeniable trend of global population aging, fall down is major outcome of sarcopenia, frailty, and multimorbidity in older adults ${ }^{1)}$ and a major roadblock to healthy aging with an independent lifestyle ${ }^{2)}$. Moreover, fall down has been an important culprit in the creation of socioeconomic burdens due to their adverse consequences requiring orthopedic surgery, rehabilitation, and care needs for geriatric syndromes and functional impairments following the initial events. Therefore, the clinical importance of screening people for high risk of falls by measuring physical performance in older people has been ever increasing ${ }^{3)}$, and the article by Kim et al. ${ }^{4)}$ is a timely examination of this issue for the field of geriatrics.

In this recent article, Kim et al. ${ }^{4)}$ showed that reaction time measured with a device using a touchscreen user interface correlates with every component of the Short Physical Performance Battery (SPPB) ${ }^{5)}$ and the Berg Balance Test $(B B S)^{6}$. As the reaction process of the protocol includes steps of perception, central processing of the information, and execution with resultant physical movement of the limb, the nature of the reaction time covers every facet of SPPB or BBS, making the correlation between the reaction time with the new device and SPPB or BBS plausible. Although the present study did not measure hard outcomes such as incidence of falls or functional impairment, we may infer the outcome relevance of this tool, taking into account a

Hee-Won Jung

Graduate School of Medical Science and Engineering, Korea Advanced Institute of Science and Technology (KAIST), Daejeon, Korea

Corresponding Author: Hee-Won Jung, MD, MSc Graduate School of Medical Science and Engineering, Korea Advanced Institute of Science and Technology (KAIST), 291, Daehak-ro, Yuseong-gu, Daejeon, 34141, Korea

Tel: +82-42-350-4283, Fax: +82-42-350-4269

E-mail: dr.ecsta@gmail.com

Received: November 16, 2017

Revised: November 16, 2017

Accepted: November 22, 2017 previous report showing associations between finger tap reaction time and functional outcomes ${ }^{7)}$.

As a recent review in the journal suggested, smart medical devices will gradually become a part of the everyday life of older people, with the current wave of the so-called 'fourth industrial revolution,8). Although previous literature showed that currently available smart healthcare devices are not quite yet ready for real-world application in terms of technological readiness ${ }^{9)}$, improving technologies with ubiquitously accessible information and communications technology will eventually bring these devices into the geriatric field. Subsequently, requirements to evaluate the efficacy, reliability, and safety of these new smart medical devices will skyrocket in the research field of geriatric medicine. In these circumstances, I hope that the Annals of Geriatric Medicine and Research, as the leading journal of clinical geriatrics in Korea, becomes the leading platform for the development and validation of novel smart healthcare devices for older adults.

Conflicts of Interest Disclosures: The researchers claim no conflicts of interest.

\section{Acknowledgements}

Dr. Hee-Won Jung was supported by the Global PhD Fellowship Program through the National Research Foundation of Korea funded by the Ministry of Education (NRF-2015H1A2A 1030117), and the 2016 Research Fund of the Korean Geriatric Society.

\section{REFERENCES}

1. Lim JY. Sarcopenia: an emerging giant greater than osteoporosis. Ann Geriatr Med Res 2016;20:167.

2. Kim SK, Jeong KY, Lee JS, Choi HS, Hong HP, Ko YG. Risk of traumatic intracranial hemorrhage from low-energy falls in the oldest-old patients. Ann Geriatr Med Res 2016;20:221-8.

3. Jang IY, Jung HW, Lee CK, Lee YS, Lee E, Kim DH. Comparison between Korean Version of Physical Activity Scale for the Elderly and International Physical Activity Questionnaire-Short Form in evaluation of frailty phenotype. Ann Geriat Med Res 2017;21: 101-7. 
4. Kim B, Won CW, Min JY, Kim S, Kim M, Kim BS, et al. Association between computerized reaction time, Short Physical Performance Battery and Berg Balance Scale in the communitydwelling older adults. Ann Geriat Med Res 2017;21:108-14.

5. Guralnik JM, Simonsick EM, Ferrucci L, Glynn RJ, Berkman LF, Blazer DG, et al. A short physical performance battery assessing lower extremity function: association with self-reported disability and prediction of mortality and nursing home admission. J Gerontol 1994;49:M85-94.

6. Bogle Thorbahn LD, Newton RA. Use of the Berg Balance Test to predict falls in elderly persons. Phys Ther 1996;76:576-83.
7. Shim JS, Kim KI, Lim JY, Kim KW, Kim WS, Paik NJ. Finger tap reaction time as an independent prognostic factor for functional outcome in older adults. Ann Geriat Med Res 2017;21: 64-9.

8. Lee JY, Lim JY. The prospect of the fourth industrial revolution and home healthcare in super-aged society. Ann Geriat Med Res 2017;21:95-100.

9. Liu L, Stroulia E, Nikolaidis I, Miguel-Cruz A, Rios Rincon A. Smart homes and home health monitoring technologies for older adults: a systematic review. Int J Med Inform 2016;91:44-59. 